\title{
Approximate $m$-Lie homomorphisms and approximate Jordan $m$-Lie homomorphisms associated to a parametric additive functional equation
}

\author{
Hassan Azadi Kenary ${ }^{1}$, Hamid Rezaei ${ }^{2}$, Madjid Eshaghi Gordji ${ }^{3}$, Choonkil Park ${ }^{4^{*}}$ and Sang Og Kim ${ }^{5}$
}

\footnotetext{
* Correspondence: baak@hanyang. ac.kr

${ }^{4}$ Research Institute for Natural Sciences, Hanyang University, Seoul 133-791, Korea

Full list of author information is available at the end of the article
}

\begin{abstract}
Using fixed point method, we establish the Hyers-Ulam stability of $m$-Lie homomorphisms and Jordan $m$-Lie homomorphisms in $m$-Lie algebras associated to the following generalized Jensen functional equation

$$
\sum_{i=1}^{m} \mu f\left(x_{i}\right)=\frac{1}{2 m}\left[\sum_{i=1}^{m} f\left(\mu m x_{i}+\sum_{j=1, i \neq j}^{m} x_{j}\right)+f\left(\sum_{i=1}^{m} \mu x_{i}\right)\right]
$$

for a fixed positive integer $m$ with $m \geq 2$.

Mathematics Subject Classification (2010): Primary 17A42, 39B82, $39 B 52$.

Keywords: m-Lie algebra, homomorphism, Jordan homomorphism, Hyers-Ulam stability, fixed point approach, Jensen-type functional equation
\end{abstract}

\section{Introduction}

Let $n$ be a natural number greater or equal to 3 . The notion of an $n$-Lie algebra was introduced by V.T. Filippov in 1985 [1]. The Lie product is taken between $n$ elements of the algebra instead of two. This new bracket is $n$-linear, anti-symmetric and satisfies a generalization of the Jacobi identity. For $n=3$, this product is a special case of the Nambu bracket, well-known in physics, which was introduced by Nambu [2] in 1973, as a generalization of the Poisson bracket in Hamiltonian mechanics.

An $n$-Lie algebra is a natural generalization of a Lie algebra. Namely:

A vector space $V$ together with a multi-linear, antisymmetric $n$-ary operation [ ]: $\Lambda^{n} V \rightarrow V$ is called an $n$-Lie algebra, $n \geq 3$, if the $n$-ary bracket is a derivation with respect to itself, i.e.,

$$
\left[\left[x_{1}, \cdots, x_{n}\right], x_{n+1}, \cdots, x_{2 n-1}\right]=\sum_{i=1}^{n}\left[x_{1}, \cdots, x_{i-1}\left[x_{i}, x_{n+1}, \cdots, x_{2 n-1}\right], \cdots, x_{n}\right]
$$

where $x_{1}, x_{2}, \cdots, x_{2 n-1} \in V$. The equation (1.1) is called the generalized Jacobi identity. The meaning of this identity is similar to that of the usual Jacobi identity for a Lie algebra (which is a 2-Lie algebra).

In [1] and several subsequent papers [3-5], a structure theory of finite-dimensional $n$-Lie algebras over a field $\mathbb{F}$ of characteristic 0 was developed.

(C) 2012 Kenary et al; licensee Springer. This is an Open Access article distributed under the terms of the Creative Commons Attribution License (http://creativecommons.org/licenses/by/2.0), which permits unrestricted use, distribution, and reproduction in any medium, provided the original work is properly cited. 
$n$-ary algebras have been considered in physics in the context of Nambu mechanics $[2,6]$ and, recently (for $\mathrm{n}=3$ ), in the search for the effective action of coincident $M 2$-branes in $M$-theory initiated by the Bagger-Lambert-Gustavsson (BLG) model [7,8] (further references on the physical applications of $n$-ary algebras are given in [9]).

From now on, we only consider $n$-Lie algebras over the field of complex numbers. An $n$-Lie algebra $A$ is a normed $n$-Lie algebra if there exists a norm \|\| on $A$ such that $\left\|\left[x_{1}, x_{2}, \cdots, x_{n}\right]\right\| \leq\left\|x_{1}\right\|\left\|x_{2}\right\| \cdots\left\|x_{n}\right\|$ for all $x_{1}, x_{2}, \cdots, x_{n} \in A$. A normed $n$-Lie algebra $A$ is called a Banach $n$-Lie algebra if $(A,\|\|)$ is a Banach space.

Let $\left(A,[]_{A}\right)$ and $\left(B,[]_{B}\right)$ be two Banach $n$-Lie algebras. A $\mathbb{C}$-linear mapping $H$ : $(A$, []$\left._{A}\right) \rightarrow\left(B,[]_{B}\right)$ is called an $n$-Lie homomorphism if

$$
H\left(\left[x_{1} x_{2} \cdots x_{n}\right]_{A}\right)=\left[H\left(x_{1}\right) H\left(x_{2}\right) \cdots H\left(x_{n}\right)\right]_{B}
$$

for all $x_{1}, x_{2}, \cdots, x_{n} \in A$. A $\mathbb{C}$-linear mapping $H:\left(A,[]_{A}\right) \rightarrow\left(B,[]_{B}\right)$ is called a Jordan $n$-Lie homomorphism if

$$
H\left([x x \cdots x]_{A}\right)=[H(x) H(x) \cdots H(x)]_{B}
$$

for all $x \in A$.

The study of stability problems had been formulated by Ulam [10] during a talk in 1940: Under what condition does there exist a homomorphism near an approximate homomorphism? In the following year, Hyers [11] was answered affirmatively the question of Ulam for Banach spaces, which states that if $\varepsilon>0$ and $f: X \rightarrow Y$ is a mapping with $X$ a normed space and $Y$ a Banach spaces such that

$$
\|f(x+y)-f(x)-f(y)\| \leq \varepsilon
$$

for all $x, y \in X$, then there exists a unique additive map $T: X \rightarrow Y$ such that

$$
\|f(x)-T(x)\| \leq \varepsilon
$$

for all $x \in X$. A generalized version of the theorem of Hyers for approximately linear mappings was presented by Rassias [12] in 1978 by considering the case when inequality (1.2) is unbounded.

In 2003, Cădariu and Radu applied the fixed point method to the investigation of the Jensen functional equation [13] (see also [14-16]). They could present a short and a simple proof (different of the "direct method ", initiated by Hyers in 1941) for the Hyers-Ulam stability of Jensen functional equation [13] and for quadratic functional equation [14].

Park and Rassias [17] proved the stability of homomorphisms in $C^{*}$-algebras and Lie $C^{*}$ algebras and also of derivations on $C^{*}$-algebras and Lie $C^{*}$-algebras for the Jensen-type functional equation

$$
\mu f\left(\frac{x+y}{2}\right)+\mu f\left(\frac{x-y}{2}\right)-f(\mu x)=0
$$

for all $\mu \in \mathbb{T}^{1}:=\{\lambda \in \mathbb{C}:|\lambda|=1\}$.

In this paper, by using fixed point method, we establish the Hyers-Ulam stability of $n$-Lie homomorphisms and Jordan $n$-Lie homomorphisms in $n$-Lie Banach algebras associated to the following generalized Jensen-type functional equation 


$$
\sum_{i=1}^{m} \mu f\left(x_{i}\right)-\frac{1}{2 m}\left[\sum_{i=1}^{m} f\left(\mu m x_{i}+\sum_{j=1, i \neq j}^{m} \mu x_{j}\right)+f\left(\sum_{i=1}^{m} \mu x_{i}\right)\right]=0
$$

for all

$$
\mu \in \mathbb{T}_{\frac{1}{n_{o}}}^{1}:=\left\{e^{i \theta}: 0 \leq \theta \leq \frac{2 \pi}{n_{o}}\right\} \cup\{1\}
$$

where $m \geq 2$.

Throughout this paper, assume that $\left(A,[]_{A}\right)$ and $\left(B,[]_{B}\right)$ are two $m$-Lie Banach algebras.

\section{Main results}

Before proceeding to the main results, we recall a fundamental result in fixed point theory.

Theorem 2.1. [18]Let $(\Omega, d)$ be a complete generalized metric space and $T: \Omega \rightarrow \Omega$ be a strictly contractive function with Lipschitz constant L. Then for each given $x \in \Omega$, either

$$
d\left(T^{m} x, T^{m+1} x\right)=\infty \text { for all } m \geq 0
$$

or other exists a natural number $m_{0}$ such that

- $d\left(T^{m} x, T^{m+1} x\right)<\infty$ for all $m \geq m_{0}$;

- the sequence $\left\{T^{m} x\right\}$ is convergent to a fixed point $y^{*}$ of $T$;

- $y^{*}$ is the unique fixed point of $T$ in $\Lambda=\left\{y \in \Omega: d\left(T^{m 0} x, y\right)<\infty\right\}$;

- $d\left(y, y^{*}\right) \leq \frac{1}{1-L} d(y, T y)$ for all $y \in \Lambda$.

Theorem 2.2. Let $V$ and $W$ be real vector spaces. $A$ mapping $f: V \rightarrow W$ satisfies the following functional equation

$$
\sum_{i=1}^{m} f\left(x_{i}\right)=\frac{1}{2 m}\left[\sum_{i=1}^{m} f\left(m x_{i}+\sum_{j=1, i \neq j}^{m} x_{j}\right)+f\left(\sum_{i=1}^{m} x_{i}\right)\right]
$$

if and only $f f$ is additive.

Proof. It is easy to prove the theorem. $\square$

We start our work with the main theorem of the our paper.

Theorem 2.3. Let $n_{0} \in \mathbb{N}$ be a fixed positive integer. Let $f: A \rightarrow B$ be a mapping for which there exists a function $\varphi: A^{m} \rightarrow[0, \infty)$ such that

$$
\begin{aligned}
& \left\|\mu \sum_{i=1}^{m} \mu f\left(x_{i}\right)-\frac{1}{2 m}\left[\sum_{i=1}^{m} f\left(\mu m x_{i}+\sum_{j=1, i \neq j}^{m} \mu x_{j}\right)+f\left(\sum_{i=1}^{m} \mu x_{i}\right)\right]\right\| \\
& \quad \leq \varphi\left(x_{1}, x_{2}, \cdots, x_{m}\right) \\
& \left\|f\left(\left[x_{1} x_{2} \cdots x_{n}\right]_{A}\right)-\left[f\left(x_{1}\right) f\left(x_{2}\right) \cdots f\left(x_{m}\right)\right]_{B}\right\| \leq \varphi\left(x_{1}, x_{2}, \cdots, x_{m}\right)
\end{aligned}
$$


for all $\mu \in \mathbb{T}_{\frac{1}{n_{0}}}^{1}$ and all $x_{1}, \cdots, x_{m} \in A$. If there exists an $L<1$ such that

$$
\varphi\left(x_{1}, x_{2}, \ldots, x_{m}\right) \leq m L \varphi\left(\frac{x_{1}}{m}, \frac{x_{2}}{m}, \cdots, \frac{x_{m}}{m}\right)
$$

for all $x_{1}, \cdots, x_{m} \in A$, then there exists a unique m-Lie homomorphism $H: A \rightarrow B$ such that

$$
\|f(x)-H(x)\| \leq \frac{\varphi(x, 0,0, \cdots, 0)}{m-m L}
$$

for all $x \in A$.

Proof. Let $\Omega$ be the set of all functions from $A$ into $B$ and let

$$
d(g, h):=\inf \left\{C \in \mathbb{R}^{+}:\|g(x)-h(x)\|_{B} \leq C \phi(x, 0, \cdots, 0), \forall x \in A\right\} .
$$

It is easy to show that $(\Omega, d)$ is a generalized complete metric space [19].

Now we define the mapping J: $\Omega \rightarrow \Omega$ by

$$
J(h)(x)=\frac{1}{m} h(m x)
$$

for all $x \in A$.

Note that for all $g, h \in \Omega$,

$$
\begin{aligned}
d(g, h)<C & \Rightarrow\|g(x)-h(x)\| \leq C \phi(x, 0, \cdots, 0) \\
& \Rightarrow\left\|\frac{1}{m} g(m x)-\frac{1}{m} h(m x)\right\| \leq \frac{C \varphi(m x, 0, \cdots, 0)}{|m|^{\ell}} \\
& \Rightarrow\left\|\frac{1}{m} g(m x)-\frac{1}{m} h(m x)\right\| \leq L C \varphi(x, 0, \cdots, 0) \\
& \Rightarrow d(J(g), J(h)) \leq L C
\end{aligned}
$$

for all $x \in A$. Hence we see that

$$
d(J(g), J(h)) \leq L d(g, h)
$$

for all $g, h \in \Omega$. It follows from (2.3) that

$$
\lim _{k \rightarrow \infty} \frac{\varphi\left(m^{k} x_{1}, m^{k} x_{2}, \cdots, m^{k} x_{m}\right)}{m^{k}} \leq \lim _{k \rightarrow \infty} L^{k} \varphi\left(x_{1}, \cdots, x_{m}\right)=0
$$

for all $x_{1}, \cdots, x_{m} \in A$. Putting $\mu=1, x_{1}=x$ and $x_{j}=0(j=2, \cdots, n)$ in (2.1), we obtain

$$
\left\|\frac{f(m x)}{m}-f(x)\right\| \leq \frac{\varphi(x, 0, \cdots, 0)}{m}
$$

for all $x \in A$. Therefore,

$$
d(f, J(f)) \leq \frac{1}{m}<\infty .
$$

By Theorem 2.1, $J$ has a unique fixed point in the set $X_{1}:=\{h \in \Omega: d(f, h)<\infty\}$. Let $H$ be the fixed point of $J$. $H$ is the unique mapping with 


$$
H(m x)=m H(x)
$$

such that there exists $C \in(0, \infty)$ satisfying

$$
\|f(x)-H(x)\| \leq C \varphi(x, 0, \cdots, 0)
$$

for all $x \in A$. On the other hand, we have $\lim _{k \rightarrow \infty} d\left(J^{k}(f), H\right)=0$ and so

$$
\lim _{k \rightarrow \infty} \frac{1}{m^{k}} f\left(m^{k} x\right)=H(x)
$$

for all $x \in A$. By Theorem 2.1, we have

$$
d(f, H) \leq \frac{1}{1-L} d(f, J(f))
$$

It follows from (2.6) and (2.8) that

$$
d(f, H) \leq \frac{1}{m-m L} .
$$

This implies the inequality (2.4). By (2.2), we have

$$
\begin{aligned}
& \left\|H\left(\left[x_{1} x_{2} \cdots x_{m}\right]_{A}\right)-\left[H\left(x_{1}\right) H\left(x_{2}\right) H\left(x_{3}\right) \cdots H\left(x_{m}\right)\right]_{B}\right\| \\
& =\lim _{k \rightarrow \infty}\left\|\frac{H\left(\left[m^{k} x_{1} m^{k} x_{2} \cdots m^{k} x_{m}\right]_{A}\right)}{m^{m k}}-\frac{\left(\left[H\left(m^{k} x_{1}\right) H\left(m^{k} x_{2}\right) H\left(m^{k} x_{3}\right) \cdots H\left(m^{k} x_{m}\right)\right]_{B}\right)}{m^{m k}}\right\| \\
& \leq \lim _{m \rightarrow \infty} \frac{\varphi\left(m^{k} x_{1}, m^{k} x_{2}, \cdots, m^{k} x_{m}\right)}{m^{m k}}=0
\end{aligned}
$$

for all $x_{1}, \cdots, x_{m} \in A$. Hence

$$
H\left(\left[x_{1} x_{2} \cdots x_{m}\right]_{A}\right)=\left[H\left(x_{1}\right) H\left(x_{2}\right) H\left(x_{3}\right) \cdots H\left(x_{m}\right)\right]_{B}
$$

for all $x_{1}, \cdots, x_{m} \in A$.

On the other hand, it follows from (2.1), (2.5) and (2.7) that

$$
\begin{aligned}
& \left\|\sum_{i=1}^{m} H\left(x_{i}\right)-\frac{1}{2 m}\left[\sum_{i=1}^{m} H\left(m x_{i}+\sum_{j=1, i \neq j}^{m} x_{j}\right)+H\left(\sum_{i=1}^{m} x_{i}\right)\right]\right\|_{B} \\
& =\lim _{k \rightarrow \infty} \frac{1}{m^{k}}\left\|\sum_{i=1}^{m} f\left(m^{k} x_{i}\right)-\frac{1}{2 m}\left[\sum_{i=1}^{m} f\left(m^{k+1} x_{i}+\sum_{j=1, i \neq j}^{m} m^{k} x_{j}\right)+f\left(\sum_{i=1}^{m} m^{k} x_{i}\right)\right]\right\| \\
& \leq \lim _{m \rightarrow \infty} \frac{\varphi\left(m^{k} x_{1}, m^{k} x_{2}, \cdots, m^{k} x_{m}\right)}{m^{k}}=0
\end{aligned}
$$

for all $x_{1}, \cdots, x_{m} \in A$. Then

$$
\sum_{i=1}^{m} H\left(x_{i}\right)=\frac{1}{2 m}\left[\sum_{i=1}^{m} H\left(m x_{i}+\sum_{j=1, i \neq j}^{m} x_{j}\right)+H\left(\sum_{i=1}^{m} x_{i}\right)\right]
$$

for all $x_{1}, \cdots, x_{m} \in A$. So by Theorem 2.1, $H$ is additive. Letting $x_{i}=x$ for all $i=1$, $2, \cdots, n$ in (2.1), we obtain

$$
\|\mu f(x)-f(\mu x)\| \leq \varphi(x, x, \cdots, x)
$$


for all $x \in A$. It follows that

$$
\begin{aligned}
\|H(\mu x)-\mu H(x)\| & =\lim _{k \rightarrow \infty} \frac{\left\|f\left(\mu m^{k} x\right)-\mu f\left(m^{k} x\right)\right\|}{m^{k}} \\
& \leq \lim _{k \rightarrow \infty} \frac{\varphi\left(m^{k} x, m^{k} x, \cdots, m^{k} x\right)}{m^{k}}=0
\end{aligned}
$$

for all $\mu \in \mathbb{T}_{\frac{1}{n_{0}}}^{1}$ and all $x \in A$. One can show that the mapping $H: A \rightarrow B$ is $\mathbb{C}$ -linear.

Hence $H: A \rightarrow B$ is an $m$-Lie homomorphism satisfying (2.4), as desired. $\square$

Corollary 2.4. Let $\theta$ and $p$ be nonnegative real numbers such that $p<1$. Suppose that a mapping $f: A \rightarrow B$ satisfies

$$
\begin{aligned}
& \left\|\mu \sum_{i=1}^{m} \mu f\left(x_{i}\right)-\frac{1}{2 m}\left[\sum_{i=1}^{m} f\left(\mu m x_{i}+\sum_{j=1, i \neq j}^{m} \mu x_{j}\right)+f\left(\sum_{i=1}^{m} \mu x_{i}\right)\right]\right\| \leq \theta \sum_{i=1}^{m}\left(\left\|x_{i}\right\|^{p}\right), \\
& \left\|f\left(\left[x_{1} x_{2} \cdots x_{n}\right]_{A}\right)-\left[f\left(x_{1}\right) f\left(x_{2}\right) \cdots f\left(x_{m}\right)\right]_{B}\right\| \leq \theta \sum_{i=1}^{m}\left(\left\|x_{i}\right\|^{p}\right)
\end{aligned}
$$

for all $\mu \in \mathbb{T}_{\frac{1}{n_{0}}}^{1}$ and all $x_{1}, \cdots, x_{m} \in A$. Then there exists a unique $m$-Lie homomorphism $H: A \rightarrow B$ such that

$$
\|f(x)-H(x)\| \leq \frac{\theta\|x\|^{p}}{\left(m-m^{p}\right)}
$$

for all $x \in A$.

Proof. Putting $\varphi\left(x_{1}, x_{2}, \cdots, x_{m}\right):=\theta \sum_{i=1}^{m}\left(\left\|x_{i}\right\|^{p}\right)$ for all $x_{1}, \cdots, x_{n} \in A$ and letting $L=m^{p-1}$ in Theorem 2.3, we obtain (2.11).

Similarly, we have the following and we will omit the proof.

Theorem 2.5. Let $f: A \rightarrow B$ be a mapping for which there exists a function $\phi: A^{m} \rightarrow$ $[0, \infty)$ satisfying (2.1) and (2.2). If there exists an $L<1$ such that

$$
\varphi\left(\frac{x_{1}}{m}, \frac{x_{2}}{m}, \ldots, \frac{x_{m}}{m}\right) \leq \frac{L}{m} \varphi\left(x_{1}, x_{2}, \ldots, x_{m}\right)
$$

for all $x_{1}, \cdots, x_{m} \in A$, then there exists a unique m-Lie homomorphism $H: A \rightarrow B$ such that

$$
\|f(x)-H(x)\| \leq \frac{L \varphi(x, 0,0, \cdots, 0)}{m-m L}
$$

for all $x \in A$.

Corollary 2.6. Let $\theta$ and $p$ be nonnegative real numbers such that $p>1$. Suppose that a mapping $f: A \rightarrow B$ satisfies (2.9) and (2.10). Then there exists a unique $m$-Lie homomorphism $H: A \rightarrow B$ such that

$$
\|f(x)-H(x)\| \leq \frac{m \theta\|x\|^{p}}{m^{p+1}-m^{2}}
$$


for all $x \in A$.

Proof. Putting $\varphi\left(x_{1}, x_{2}, \cdots, x_{m}\right):=\theta \sum_{i=1}^{m}\left(\left\|x_{i}\right\|^{p}\right)$ for all $x_{1}, \cdots, x_{n} \in A$ and letting $L=m^{1-p}$ in Theorem 2.5, we obtain (2.12).

Theorem 2.7. Let $n_{0} \in \mathbb{N}$ be a fixed positive integer. Let $f: A \rightarrow B$ be a mapping for which there exists a function $\phi: A^{n} \rightarrow[0, \infty)$ such that

$$
\begin{gathered}
\left\|\mu \sum_{i=1}^{m} \mu f\left(x_{i}\right)-\frac{1}{2 m}\left[\sum_{i=1}^{m} f\left(\mu m x_{i}+\sum_{j=1, i \neq j}^{m} \mu x_{j}\right)+f\left(\sum_{i=1}^{m} \mu x_{i}\right)\right]\right\| \\
\leq \varphi\left(x_{1}, x_{2}, \cdots, x_{m}\right),
\end{gathered}
$$

for all $\mu \in \mathbb{T}_{\frac{1}{n_{0}}}^{1}$ and all $x_{1}, \cdots, x_{m} \in A$. If there exists an $L<1$ such that

$$
\varphi\left(x_{1}, x_{2}, \cdots, x_{m}\right) \leq m L \varphi\left(\frac{x_{1}}{m}, \frac{x_{2}}{m}, \cdots, \frac{x_{m}}{m}\right)
$$

for all $x_{1}, \cdots, x_{m} \in A$, then there exists a unique Jordan m-Lie homomorphism $H: A$ $\rightarrow B$ such that

$$
\|f(x)-H(x)\| \leq \frac{\varphi(x, 0, \cdots, 0)}{m-m L}
$$

for all $x \in A$.

Proof. By the same reasoning as in the proof of Theorem 2.3, we can define the mapping

$$
H(x)=\lim _{k \rightarrow \infty} \frac{1}{m^{k}} f\left(m^{k} x\right)
$$

for all $x \in A$. Moreover, we can show that $H$ is $\mathbb{C}$-linear. By (2.14), we get that

$$
\begin{aligned}
& \left\|H\left([x x \cdots x]_{A}\right)-[H(x) H(x) \cdots H(x)]_{B}\right\| \\
& =\lim _{k \rightarrow \infty} \| \frac{1}{m^{m k}} H\left(\left[m^{k} x \cdots m^{k} x\right]_{A}\right)-\frac{1}{m^{m k}}\left(\left[H\left(m^{k} x\right) H\left(m^{k} x\right) \cdots H\left(m^{k} x\right)\right]_{B} \|\right. \\
& \leq \lim _{k \rightarrow \infty} \frac{1}{m^{m k}} \varphi\left(m^{k} x, m^{k} x, \ldots, m^{k} x\right)=0
\end{aligned}
$$

for all $x \in A$. So

$$
H\left([x x \cdots x]_{A}\right)=[H(x) H(x) \cdots H(x)]_{B}
$$

for all $x \in A$. Hence $H: A \rightarrow B$ is a Jordan $m$-Lie homomorphism satisfying (2.15).

Corollary 2.8. Let $\theta$ and $p$ be nonnegative real numbers such that $p<1$. Suppose that a mapping $f: A \rightarrow B$ satisfies

$$
\begin{aligned}
& \left\|\mu \sum_{i=1}^{m} \mu f\left(x_{i}\right)-\frac{1}{2 m}\left[\sum_{i=1}^{m} f\left(\mu m x_{i}+\sum_{j=1, i \neq j}^{m} \mu x_{j}\right)+f\left(\sum_{i=1}^{m} \mu x_{i}\right)\right]\right\| \leq \theta \sum_{i=1}^{n}\left(\left\|x_{i}\right\|^{p}\right), \\
& \left\|f\left([x x \cdots x]_{A}\right)-[f(x) f(x) \cdots f(x)]_{B}\right\| \leq n \theta\left(\|x\|^{p}\right)
\end{aligned}
$$


for all $\mu \in \mathbb{T}_{\frac{1}{n_{0}}}^{1}$ and all $x_{1}, \cdots, x_{m} \in$ A. Then there exists a unique Jordan $m$-Lie homomorphism $H: A \rightarrow B$ such that

$$
\|f(x)-H(x)\| \leq \frac{\theta\|x\|^{p}}{m-m^{p}}
$$

for all $x \in A$.

Proof. The proof follows from Theorem 2.7 by putting $\varphi\left(x_{1}, x_{2}, \ldots, x_{m}\right):=\theta \sum_{i=1}^{m}\left(|| x_{i} \|^{p}\right)$ for all $x_{1}, \cdots, x_{m} \in A$ and letting $L=m^{p-1}$. $\square$

Similarly, we have the following and we will omit the proof.

Theorem 2.9. Let $f: A \rightarrow B$ be a mapping for which there exists a function $\phi: A^{m} \rightarrow$ $[0, \infty)$ satisfying (2.13) and (2.14). If there exists an $L<1$ such that

$$
\varphi\left(\frac{x_{1}}{m}, \frac{x_{2}}{m}, \ldots, \frac{x_{m}}{m}\right) \leq \frac{L}{m} \varphi\left(x_{1}, x_{2}, \ldots, x_{m}\right)
$$

for all $x_{1}, \cdots, x_{m} \in A$, then there exists a unique Jordan m-Lie homomorphism $H: A \rightarrow B$ such that

$$
\|f(x)-H(x)\| \leq \frac{L \varphi(x, 0,0, \cdots, 0)}{m-m L}
$$

for all $x \in A$.

Corollary 2.10. Let $\theta$ and $p$ be nonnegative real numbers such that $p>1$. Suppose that a mapping $f: A \rightarrow B$ satisfies (2.16) and (2.17). Then there exists a unique Jordan m-Lie homomorphism $H: A \rightarrow B$ such that

$$
\|f(x)-H(x)\|_{B} \leq \frac{\theta\|x\|^{p}}{m^{p}-m}
$$

for all $x \in A$.

Proof. Putting $\varphi\left(x_{1}, x_{2}, \cdots, x_{m}\right):=\theta \sum_{i=1}^{m}\left(\left\|x_{i}\right\|^{p}\right)$ for all $x_{1}, \cdots, x_{n} \in A$ and letting $L=m^{1-p}$ in Theorem 2.9, we obtain (2.18).

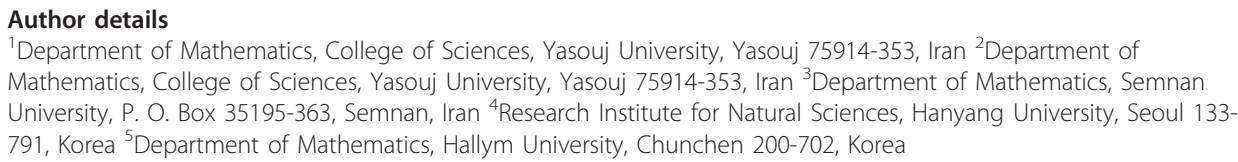


6. Takhtajan, L: On foundation of the generalized Nambu mechanics. Comm Math Phys. 160, 295-316 (1994). doi:10.1007/ BF02103278

7. Bagger, J, Lambert, N: Comments nn multiple M2-branes. J High Energy Phys 2008, 15 (2008). Art. ID 105

8. Gustavsson, A: One-loop corrections to Bagger-Lambert theory. Nuclear Phys B. 807, 315-333 (2009). doi:10.1016/j. nuclphysb.2008.09.003

9. Azcarraga, JA, Izquierdo, JM: $n$-ary algebras: a review with applications. J Phys A 43, 1-117 (2010). Art. ID 293001

10. Ulam, SM: A Collection of Mathematical Problems. Interscience Tracts in Pure and Applied Mathematics. Interscience Publ. New York-London (1940)

11. Hyers, DH: On the stability of the linear functional equation. Proc Natl Acad Sci. 27, 222-224 (1941). doi:10.1073/ pnas.27.4.222

12. Rassias, TM: On the stability of the linear mapping in Banach spaces. Proc Amer Math Soc. 72, 297-300 (1978). doi:10.1090/50002-9939-1978-0507327-1

13. Cădariu, L, Radu, V: Fixed points and the stability of Jensen's functional equation. J Inequal Pure Appl Math 4 (2003). No. 1, Art. 4

14. Cădariu, L, Radu, V: Fixed points and the stability of quadratic functional equations. An Univ Timişoara Ser Mat Inform. 41, 25-48 (2003)

15. Eshaghi Gordji, M, Ghaemi, MB, Kaboli Gharetapeh, S, Shams, S, Ebadian, A: On the stability of J*-derivations. J Geom Phys. 60, 454-459 (2010). doi:10.1016/.geomphys.2009.11.004

16. Eshaghi Gordji, M, Najati, A: Approximately J*-homomorphisms: a fixed point approach. J Geom Phys. 60, 809-814 (2010). doi:10.1016/j.geomphys.2010.01.012

17. Park, C, Rassias, JM: Stability of the Jensen-type functional equation in $C^{*}$-algebras: a fixed point approach. Abstr Appl Anal 2009, 17 (2009). Art. ID 360432

18. Diaz, JB, Margolis, B: A fixed point theorem of the alternative for contractions on a generalized complete metric space. Bull Amer Math Soc. 74, 305-309 (1968). doi:10.1090/50002-9904-1968-11933-0

19. Cădariu, L, Radu, V: On the stability of the Cauchy functional equation: a fixed point approach. Grazer Mathematische Berichte. 346, 43-52 (2004)

doi:10.1186/1687-1847-2012-125

Cite this article as: Kenary et al: Approximate $m$-Lie homomorphisms and approximate Jordan $m$-Lie

homomorphisms associated to a parametric additive functional equation. Advances in Difference Equations 2012

2012:125.

\section{Submit your manuscript to a SpringerOpen ${ }^{\odot}$} journal and benefit from:

- Convenient online submission

- Rigorous peer review

- Immediate publication on acceptance

- Open access: articles freely available online

- High visibility within the field

- Retaining the copyright to your article

Submit your next manuscript at $\gg$ springeropen.com 